\title{
A Bright Source of High- Energy X-rays: Final Report on LDRD Project 04-FS-007
}

\author{
Jeffrey D. Colvin \& Thomas E. Felter \\ Lawrence Livermore National Laboratory
}

Peter C. Searson \& Min Chen Johns Hopkins University

February 14, 2005 


\section{Disclaimer}

This document was prepared as an account of work sponsored by an agency of the United States Government. Neither the United States Government nor the University of California nor any of their employees, makes any warranty, express or implied, or assumes any legal liability or responsibility for the accuracy, completeness, or usefulness of any information, apparatus, product, or process disclosed, or represents that its use would not infringe privately owned rights. Reference herein to any specific commercial product, process, or service by trade name, trademark, manufacturer, or otherwise, does not necessarily constitute or imply its endorsement, recommendation, or favoring by the United States Government or the University of California. The views and opinions of authors expressed herein do not necessarily state or reflect those of the United States Government or the University of California, and shall not be used for advertising or product endorsement purposes.

\section{Auspices Statement}

This work was performed under the auspices of the $U$. S. Department of Energy (DOE) by the University of California, Lawrence Livermore National Laboratory (LLNL) under Contract No. W-7405-Eng-48. The project (04-FS-007) was funded by the Laboratory Directed Research and Development Program at LLNL. 


\title{
FY04 LDRD Final Report \\ A Bright Source of High-Energy X-rays \\ LDRD Project Tracking Code: 04-FS-007 \\ Jeffrey D. Colvin, Principal Investigator \\ Thomas E. Felter, co-PI \\ Peter C. Searson, Johns Hopkins University \\ Min Chen, Johns Hopkins University
}

\begin{abstract}
We have demonstrated the feasibility of fabricating pure-metal foams via a novel four-step technique based upon ion beam lithography. In this report we discuss why and how such foams are useful as bright, high-photon-energy xray sources; the details of the fabrication technique we employed to make such foams; the results obtained; and what we plan to do in the future to improve the technique and turn the foams so fabricated into real laser targets for high-brightness, high-energy back lighting.
\end{abstract}

\section{Introduction/Background}

It has been known for some time that it may be feasible to create a bright, high-photonenergy x-ray source by heating a low-density target (like a gas or a pure-metal foam target having a density $<0.1 \%$ of solid density) with a high-power laser. ${ }^{1}$ Laser light will propagate through any plasma that has an electron density less than about $1 / 4$ the critical electron density, depositing its energy along the beam path via inverse bremsstrahlung interactions with free electrons. Thus, at sufficiently low densities, a laser beam can supersonically and volumetrically heat the material, providing a much higher x-ray conversion efficiency (XRCE) in the non-LTE plasma than what can be obtained by simply irradiating the solid material. Targets with a high XRCE may be suitable as backlighters for radiographing dense materials. Researchers at LLNL have already done XRCE experiments on high-Z gases ${ }^{2}$ and on metal-doped aerogel foams ${ }^{3}$. The big advantage of pure metals as bright backlighters is access to K-shell x-rays at higher photon energies; gas targets are limited to a few photon energies (Ar K- $\alpha$ line is $3 \mathrm{keV}$ vs $\mathrm{Zn} \mathrm{K}-\alpha$ line at $9 \mathrm{keV}$ ). A bright $9 \mathrm{keV}$ backlighter would open up myriad possibilities for exploring the dynamic behavior of dense metals under compression, ${ }^{4}$ for example, as well as imploding double-shell ICF capsules. ${ }^{5}$

Past work focused on a computational study of the feasibility of high XRCE with very-lowdensity pure-metal foam targets. The non-LTE problem presents computational difficulties. XRCE depends on initial foam density, foam absorption length, laser beam intensity, and the details of the opacity and emission processes in the non-LTE plasma. We have already produced a preliminary target design ${ }^{6}$ using the $2 \mathrm{D}$ radiation-hydrodynamics code 
$\mathrm{LASNEX}^{7}$. It was beyond the scope of the preliminary design work to optimize the basic target design, in particular, to explore the sensitivity of XRCE to initial foam density, foam size, and laser beam specifications (energy, spot size on target, pulse duration). Nonetheless, this preliminary work suggested that $>1 \%$ XRCE would be possible if pure-metal foams of density $<0.1 \%$ solid density could be fabricated. This XRCE is at least an order of magnitude higher than what can typically be achieved with full-density solid targets. ${ }^{8}$

Accordingly, the objective of this Feasibility Study was to investigate the feasibility of fabricating such a very-low-density pure-metal foam. We proposed to do this by making use of a novel four-step technique based on ion-tracking lithography.

\section{Research Activities}

We fabricated very-low-density pure-metal foams using ion-tracking lithography. The basic four-step process is as follows. First, an intersecting network of latent tracks was created in a host foil by $\mathrm{MeV}$ ions from an accelerator. Second, chemical etching dissolved the latent tracks (the chemical etch preferentially dissolves the material damaged by the ion passage through it), forming cylindrical holes approximately $10 \mathrm{~nm}$ in diameter (with the hole diameter proportional to the etch time). Third, these intersecting holes were filled with metal using electroplating. This forms an array of intersecting metal columns, a type of foam, supported in a plastic host. The last step is removal of the host material by chemical dissolution to leave a self-supporting thin sheet of pure metal foam.

We first determined that $14.8 \mathrm{MeV}$ Ar+ ions created in LLNL's Van de Graff accelerator provided the penetration depth and ion energy loss required for ion-tracking the 3micron-thick commercially obtained polycarbonate foils, which served as the host material. Some foils were tracked at beam angles normal to the surface, and some with beam angles off-normal and at either two or four azimuthal angles (the angle of the beam projected into the plane of the foil). The angles were selected so as to optimize the number of intersection points in the host foil, with the hope of leaving a self-supporting rigid structure after the final etch. Accordingly, the four-angle specimens (azimuthal angles of $0,90,180$, and 270 degrees, each at 45 degrees off normal), irradiated at a dose of $1.3 \times 10^{9} \mathrm{Ar}$ ions $/ \mathrm{cm}^{2}$, provided the greatest promise for creating metal foam structures, with density $0.6 \%$ of solid density for tracks etched to $10 \mathrm{~nm}$ diameter.

In addition, for the case mentioned above of four azimuths at 45 degrees off normal and our incident fluence, we developed simple relations for calculating the density and integrity of the foam. We characterize the latter by the average number of intersections for each metal column, $\mathbf{l}$. The density, $\rho_{\%}$, is conveniently defined as percent of the full density metal:

$$
\rho_{\%}=100\left\{1-\exp \left(-\Phi \pi \mathrm{d}^{2} / \cos \theta\right)\right\} \text {, }
$$


where $\mathrm{d}$ is the diameter of the etched tracks (in centimeters) and $\Phi$ is the number of ions per square centimeter in the beam, as measured normal to the beam, i.e. the (normal) beam fluence. For the geometry we used, $\theta=45$ degrees. For $\Phi d^{2}<<1$, the overlap of columns is negligible and the density expression reduces to

$\rho_{\%}=100 \Phi \pi \mathrm{d}^{2} / \cos \theta, \quad\left(\Phi \mathrm{d}^{2}<<1\right)$.

Of course, such a material without intersections would not hold together. A balance between density and the number of intersections per column is required.

The number of intersections, $\mathrm{l}$, is easily calculated:

$\mathrm{l}=\left(4+2^{1 / 2}\right) \mathrm{d} \Phi \mathrm{L}$,

where $\mathrm{L}$ is the thickness of the initial polycarbonate foil (in centimeters), which for this study is 3 microns. The factor of $\left(4+2^{1 / 2}\right)$ is appropriate for the geometry we used.

For track diameters $10 \mathrm{~nm}$ to $50 \mathrm{~nm}$, the density changes from $0.6 \%$ to $14 \%$ of solid density, and the average number of intersections changes from 2 to 10 . We opted in the end to etch to a larger diameter because we were concerned that two intersections per track, on average, would be too few to sustain a self-supporting foam structure. Even though the final density would be higher than what we ultimately hope to achieve, this study was aimed at simply demonstrating the feasibility of the technique, not achieving a particular density. We tracked and etched some dozen foils all together.

LLNL contracted with Professor Searson and his group at the Johns Hopkins University (JHU) in Baltimore to sputter-deposit on one side of the tracked and etched host material and then electro-deposit metal into the etched holes from the opposite side. They then did the final etch to dissolve the host polycarbonate. Finally, scanning electron microscopy (SEM) was done to characterize the final structures that were produced by this technique. We were particularly interested in seeing whether or not the intersections had any effect on blocking the electroplating downstream of the intersection point.

\section{Results/Technical Outcome}

Figure 1 shows a 20000x magnification SEM image of our 5\% density nickel foam in a polycarbonate host. In this example, the electroplating was halted just after the holes were filled. The "mushroom" ends of each column can be seen in the SEM and in the side view schematic. The schematic also shows an intersection. On average, there are four such intersections for each track. From the measured beam fluence during the ion irradiation $37^{ \pm} 6$ tracks per square micron is expected, in good agreement with the image. This indicates that track intersections do not interfere with track growth. The diameters of the nickel mushroom caps and nickel filled tracks are approximately $70 \mathrm{~nm}$ and $30 \mathrm{~nm}$, respectively. Note also that the average pore size (i.e., the spacing between the metal nano-struts) is less than one micron. This is not only a very-low-density foam, it is also a small-pore foam. On average, each nickel filled track has 4 intersections with other tracks. 


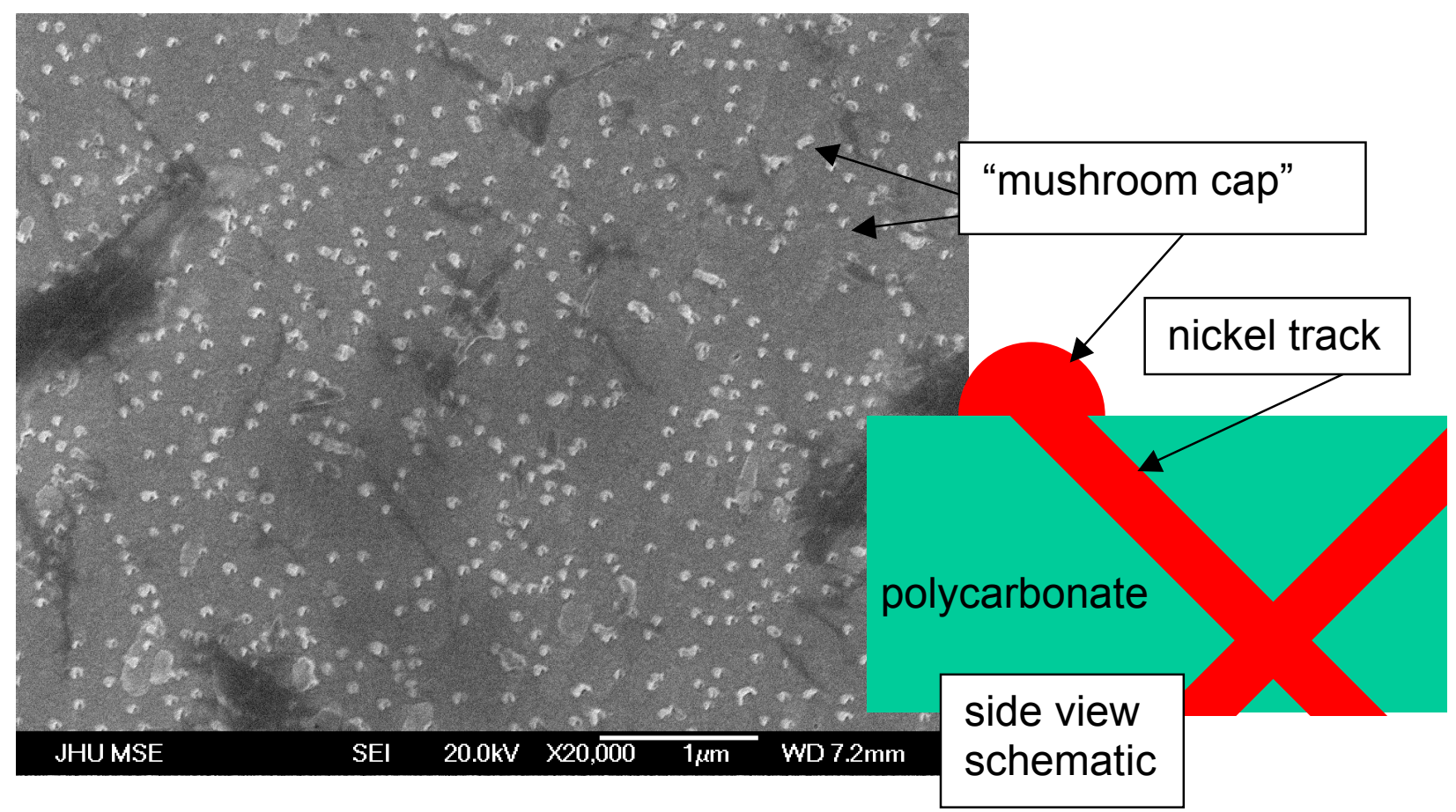

Figure 1. 20000x magnification SEM image of 5\% density nickel foam in polycarbonate host. Inset: side view schematic including intersection of two filled tracks. The electroplating was deliberately halted after the "mushroom" ends formed to ease identification of the continuously filled tracks. From a comparison of the measured beam fluence during the ion irradiation to the "mushrooms per square centimeter" measured in the SEM, we determine that most tracks fill completely and that intersections are formed. The tracks have a diameter of $30 \mathrm{~nm}$ and on average four intersections each.

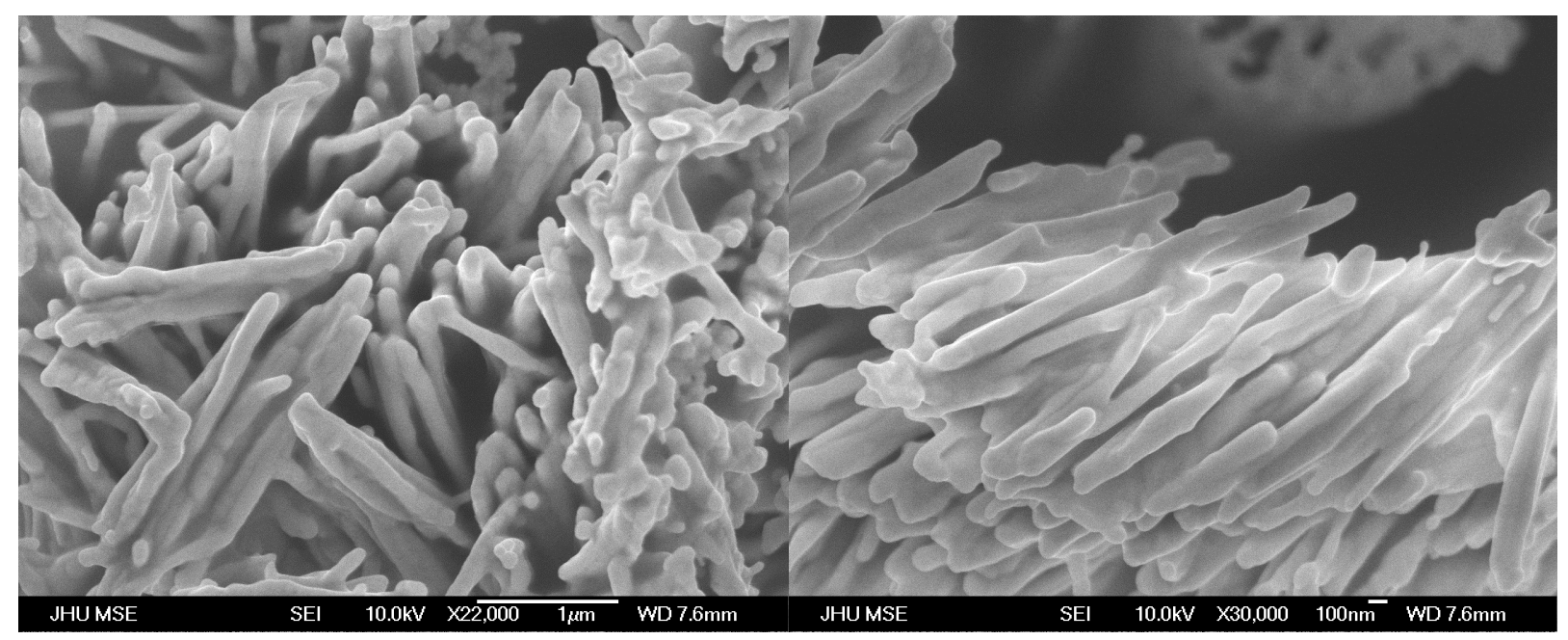

Figure 2 SEM at 22000x (left) and 30,000 (right) of gold foam after removal from polycarbonate host. Note "collapsed" structure from unmitigated capillary forces. Strong intersections and malleable struts are evident 
Figure 2 shows SEM images at 22000x (left) and 30000x (right) magnification of gold foam after removal from polycarbonate host. Note that in this case the foam "collapsed" because of capillary forces as it was extracted from the etch bath. The good news, however, is that it is quite clear that the intersections provided no obstacles to the electroplating. We were indeed successful in making an array of nano-struts as the basic components of a self-supported metal foam.

Several standard methods are being considered for extracting the foam from the host that avoids the crushing forces of surface tension. Physical methods include using a stiffer metal, e.g. platinum, and larger diameter columns. The simplest chemical method is the use of surfactants. A more sophisticated approach would exchange the etch solution with one of low surface tension. A standard recipe calls for exchange of the aqueous solution $(\mathrm{KOH})$ with acetone that in turn is exchanged for hexane. Hexane is a low surface tension organic and evaporates readily. Alternatively, the aqueous solution might be removed directly by freeze drying. Supercritical $\mathrm{CO}_{2}$ extraction is a highly effective method but technologically more challenging. Removal of the polycarbonate directly in a vacuum or hydrogen oven might be possible or in an oxygen or hydrogen plasma.

\section{Exit Plan}

We received funding for this Feasibility Study in mid-year, leaving less than six months to fabricate this novel very-low-density metal foam. We have shown that track intersections are strong and do not lead to interference during plating. In retrospect, capillary forces should have been an obvious consideration, but fortunately, mitigating the deleterious effects of these forces appears to be straightforward. Our results, though somewhat incomplete, have attracted the interest of another LDRD project at LLNL, one component of which is directed at developing x-ray imaging techniques for dynamic materials experiments driven by lasers. We are now redoing the host removal step using some of the supported foils produced in this Feasibility Study.

Once we produce high quality foams by our technique, we will pursue the fabrication of actual laser targets. We can achieve lower densities in larger 3D structures, suitable for laser targets, either by a slice-and-stack technique or a crumpling technique. ${ }^{9}$ With support from the Materials Dynamics LDRD Project (Project number 04-ERD-071) we expect to measure their X-ray conversion efficiency on the Z-Beamlet laser at the Sandia National Laboratories in Albuquerque (SNLA), in a collaboration between John Porter of SNLA and Jeff Colvin and Jim McNaney of LLNL.

\section{Acknowledgements}

The authors wish to thank Ron Musket, a private consultant to LLNL, and Larry Suter and Bruce Remington of LLNL for suggestions and encouragement.

\section{References}

1. J. Denavit and D. W. Phillion, Phys. Plasmas 1, 1971 (1994). 
2. C. A. Back, et al., Phys. Rev. Letters 87(27), 275003 (2001).

3. K. B. Fournier, et al., in Proc. SPIE, Vol. 5196 (2003).

4. B. A. Remington, et al., Met. And Mat. Trans. A 35A, 2587 (2004).

5. P. Amendt et al., Phys. Plasmas 9, 2221 (2002).

6. J. D. Colvin \& T. Felter, Bull. A.P.S. 48, 288 (2003).

7. G. B. Zimmerman and W. L. Kruer, Comments Plasma Phys. Control. Fusion 2, $51(1975)$.

8. D. L. Matthews et al., J. Appl. Phys. 54, 4260 (1983).

9. K. Matan, et al., Phys. Rev. Letters 88(7), 076101 (2002). 